\title{
Resultados Tardios do Uso Irrestrito de Stents Farmacológicos para o Tratamento do Infarto Agudo do Miocárdio com Supradesnivelamento do Segmento ST
}

\author{
Marco Aurélio de Magalhães ${ }^{1}$, Fábio Sandoli de Brito Júnior ${ }^{1}$, Breno Oliveira Almeida ${ }^{1}$, \\ Ivanise M. Gomes Amorim', Teresa Cristina Dias C. Nascimento', \\ Alexandre Antonio Cunha Abizaid ${ }^{1}$, Marco Antonio Perin ${ }^{1}$
}

\section{RESUMO}

Fundamentos: Os estudos randomizados que testaram os stents farmacológicos para o tratamento do infarto agudo do miocárdio demonstraram baixas taxas de revascularização da lesão-alvo. Entretanto, pacientes e lesões complexas foram excluídos e o seguimento tardio permanece pouco conhecido. Objetivos: Avaliar a taxa de eventos adversos combinados - óbito, reinfarto e revascularização do vasoalvo (RVA) - e a incidência de trombose em pacientes não selecionados tratados com stent farmacológico no infarto agudo do miocárdio com supradesnivelamento do segmento ST. Método: De janeiro de 2004 a dezembro de 2006, foram tratados 134 pacientes consecutivos no contexto da angioplastia primária ou de resgate. Destes, $104(77,6 \%)$ receberam ao menos um stent farmacológico. O seguimento clínico médio foi de 20,9 $\pm 10,5$ meses. Resultados: No total, 104 pacientes foram analisados, a média de idade foi de $63 \pm 12$ anos, $82 \%$ dos pacientes eram do sexo masculino, e $23 \%$ eram diabéticos. Na admissão, $17 \%$ apresentavam classificação Killip III ou IV. A taxa de sucesso angiográfico foi de $92 \%$. O tempo médio da dupla antiagregação plaquetária foi de 13,29 $\pm 9,26$ meses. A taxa cumulativa de eventos adversos combinados foi de $11,5 \%$ e a necessidade de RVA foi de $2,9 \%$. Trombose de stent definitiva ocorreu em 1,9\% dos pacientes. Na análise multivariada, o único preditor independente de eventos adversos combinados foi a classificação de Killip III ou IV à admissão (odds ratio, 9,4; intervalo de confiança de 95\%, 2,16-40,81; $p=0,003)$. Conclusões: Neste registro, composto por pacientes pertencentes ao "mundo real", o uso de stents farmacológicos no infarto agudo do miocárdio com elevação do segmento ST mostrou-se seguro e eficaz.

DESCRITORES: Infarto do miocárdio. Contenedores. Angioplastia transluminal percutânea coronária. Avaliação de resultados.

1 Hospital Israelita Albert Einstein - São Paulo, SP.

Correspondência: Marco Aurélio de Magalhães. Rua Xavier da Veiga, 81 - apto. 194 - São Paulo, SP - CEP 02021-040

Tel.: (11) 3747-0434 - Fax: (11) 3747-1035

E-mail:marcoamp@einstein.br; mademagalhaes@yahoo.com.br

Recebido em: 18/7/2007 • Aceito em: 26/9/2007
SUMMARY

Long-term Outcomes of Unrestricted Use of Drug-eluting Stents for ST-segment Elevation Myocardial Infarction

Background: Clinical trials addressing the safety and efficacy of drug-eluting stents (DES) for the treatment of patients with ST-segment elevation myocardial infarction (STEMI) showed lower rates of target vessel revascularization (TVR) compared to bare stents. However, these trials have excluded high risk patients and complex lesion subsets. Furthermore, the long-term follow-up of these patients is unknown. Objectives: To analyze the cumulative rates of major adverse events and stent thrombosis at long-term follow-up after unrestricted use of DES in patients with STEMI. Methods: From 01/2004 to 12/2006, 104 consecutive patients underwent percutaneous coronary intervention for STEMI with DES implantation and comprised the present study population. We analyzed the cumulative rates of adverse events: death, reinfarction, the target vessel revascularization (TVR) and the rates of stent thrombosis at a mean clinical follow-up of $20.9 \pm 10.5$ months. Results: A total of 104 patients were included in this study. The mean age was $63 \pm 12$ years, $82 \%$ were male and $23 \%$ diabetic. Killip III or IV were diagnosed at presentation in $17 \%$ of patients. The mean time of dual anti-platelet therapy was $13.29 \pm 9.26$ months. The cumulative incidence of major adverse events was $11.5 \%$. The incidence of TVR was $2.9 \%$. Definitive stent thrombosis occurred in $1.9 \%$ of patients. By multivariate analysis, the only independent predictor of major adverse events was Killip class III or IV at presentation (OR: $9.4 \mathrm{Cl} 95 \%$ [2.16-40.81]; p=0.003). Conclusion: In this registry composed by "real world" patients, the unrestricted use of DES for the treatment of STEMI was safe and effective at long term follow-up.

DESCRIPTORS: Myocardial infarction. Stents. Angioplasty, transluminal, percutaneous coronary. Outcome assessment. 

com Supradesnivelamento do Segmento ST. Rev Bras Cardiol Invas 2007; 15(4): 350-356.

E studos randomizados divulgados nos últimos anos sugerem que o emprego dos stents farmacológicos no infarto agudo do miocárdio determina redução significativa das taxas de reestenose $\mathrm{e}^{1-3}$. Entretanto, mais recentemente, surgiram questionamentos sobre a segurança do uso desses dispositivos no infarto agudo, por se tratar de uma condição clínica mais favorável à ocorrência de trombose dos stents ${ }^{4}$ e pela alta mortalidade reportada nesses pacientes, em caso de baixa aderência ao uso dos tienopiridínicos ${ }^{5}$. Questiona-se, também, a real necessidade de se utilizar stents farmacológicos no infarto agudo do miocárdio com o objetivo de prevenir a ocorrência de reestenose, já que as evidências indicam que as taxas de reestenose clínica, nessa situação, são baixas, mesmo quando se implantam stents convencionais ${ }^{6}$. A maioria desses dados, entretanto, deriva de estudos clínicos randomizados com número limitado de pacientes, nos quais os rígidos critérios de inclusão e os numerosos critérios de exclusão impedem que seus resultados sejam extrapolados para a prática médica diária, com pacientes e lesões de alta complexidade. Por esse motivo, decidiuse avaliar os resultados tardios do implante de stents farmacológicos em uma população não selecionada, tratada de forma consecutiva na fase aguda do infarto do miocárdio com supradesnivelamento do segmento ST por angioplastia primária ou de resgate.

\section{MÉTODO}

\section{População}

De janeiro de 2004 a dezembro de 2006, 134 pacientes consecutivos com infarto agudo do miocárdio e supradesnivelamento do segmento ST foram tratados por angioplastia primária ou de resgate, nas primeiras 12 horas do início dos sintomas. Destes, 104 (77,6\%) receberam pelo menos um stent farmacológico na artéria relacionada ao infarto, compondo a população do presente estudo. Os demais foram tratados com stents convencionais, de acordo com a decisão do operador, em geral em situações em que o diâmetro do vaso relacionado ao infarto era igual ou superior $4 \mathrm{~mm}$ ou quando a condição clínica do paciente era extremamente grave, com expectativa de sobrevivência muito pequena. Não existiram critérios clínicos ou angiográficos de exclusão e os dados foram analisados retrospectivamente quanto à taxa de eventos adversos maiores cumulativos.

\section{Definições}

Considerou-se infarto agudo do miocárdio a presença de dor torácica prolongada, associada a supradesnivelamento do segmento ST $>1 \mathrm{~mm}$ em pelo menos duas derivações contíguas ou bloqueio de ramo esquerdo agudo ou presumivelmente novo. Definiu-se choque cardiogênico (Killip IV) como hipotensão persistente (pressão arterial sistólica $<90 \mathrm{mmHg}$ por no mínimo 30 minutos) ou necessidade de utilizar drogas vasoativas.
Doença arterial coronária multiarterial foi definida como a presença de estenose angiográfica $\geq 50 \%$ em pelo menos dois vasos epicárdicos. Os eventos adversos maiores combinados foram definidos como a ocorrência de morte por qualquer causa, reinfarto ou reestenose clínica, com necessidade de nova revascularização do vaso inicialmente abordado por angioplastia ou cirurgia. Analisou-se a ocorrência de trombose de acordo com os critérios recentemente estabelecidos pelo Academic Research Consortium ${ }^{7}$, que classifica a trombose dos stents como definitiva, provável ou possível e, de acordo com o intervalo de tempo, em: precoce (0 a 30 dias), tardia (31 a 365 dias) e muito tardia (> 365 dias). Definiu-se reinfarto como a ocorrência de sintomas isquêmicos recorrentes acompanhados por alteração eletrocardiográfica ou elevação dos marcadores cardíacos em pelo menos duas vezes o valor de referência. Determinou-se a função ventricular esquerda pela ecocardiografia (equipamento Phillips HDI 5000, Phillips, Holanda), conforme normas da Sociedade Americana de Ecocardiografia ${ }^{8}$, sendo utilizada a menor fração de ejeção documentada durante a fase hospitalar do tratamento do infarto.

\section{Intervenção coronária primária}

Realizou-se o procedimento conforme técnicas previamente estabelecidas e utilizou-se o acesso femoral em todos os casos. Os pacientes receberam aspirina na admissão e 300 mg de clopidogrel na admissão ou imediatamente após a intervenção.

A escolha do stent farmacológico ocorreu a critério do operador, bem como o uso adjuvante do abciximab (ReoPro, Eli Lilly - Indianápolis, Indiana, Estados Unidos; Centocor - Horsham, Pensilvânia, Estados Unidos) e de dispositivos de trombectomia (cateter Pronto V3, Vascular Solutions - Minneapolis, Minnesota, Estados Unidos, ou X-sizer, EV3 - Plymouth, Minnesota, Estados Unidos). Administrou-se heparina não-fracionada na dose $100 \mathrm{U} / \mathrm{kg}$ ou $70 \mathrm{U} / \mathrm{kg}$, em caso de uso concomitante de abciximab. O nível de anticoagulação foi monitorado utilizando-se o tempo de coagulação ativado (TCA) medido pelo equipamento Hemocron 801. Doses adicionais de heparina foram administradas quando necessário, objetivando atingir TCA entre $250 \mathrm{~s}$ e $350 \mathrm{~s}$ ou entre $200 \mathrm{~s}$ e 300 s quando se fez uso concomitante de abciximab. Definiu-se sucesso angiográfico quando, ao final da intervenção, se obteve estenose residual < 30\% e fluxo coronário anterógrado normal (TIMI 3).

O tempo de manutenção do esquema de antiagregação plaquetária dupla dependeu da orientação do cardiologista clínico do paciente.

\section{Seguimento clínico}

As informações do seguimento clínico dos pacientes foram obtidas, retrospectivamente, por contato telefônico ou eletrônico (e-mail). Estabeleceu-se contato com 
o próprio paciente em $84,6 \%$ dos casos, com familiares em $3,8 \%$, e com o médico de referência em $9,6 \%$. A consulta de arquivos hospitalares foi a fonte dos dados em $2 \%$ dos casos.

\section{Análise angiográfica quantitativa}

Realizou-se a análise angiográfica quantitativa com o sistema validado de detecção de bordas CASS II (Pie Medical - Maastricht, Holanda) ${ }^{9}$. Nos casos em que a artéria relacionada ao infarto apresentava-se ocluída, foram utilizadas as primeiras projeções após o restabelecimento do fluxo coronário anterógrado, conseguido pela passagem do fio-guia 0,014" ou após a pré-dilatação, para medir o comprimento da lesão.

\section{Análise estatística}

As variáveis categóricas estão representadas pelo número absoluto ou porcentual (\%) e as variáveis numéricas estão representadas pela média e pelo desvio padrão ou mediana com variação interquartil. As curvas de sobrevida livre de eventos adversos e da revascularização do vaso-alvo (RVA) foram realizadas pelo método de Kaplan-Meier. A análise univariada foi utilizada para identificar os fatores relacionados à ocorrência de eventos clínicos adversos. Dentre esses fatores, aqueles com nível de significância $<0,2$ foram incluídos no modelo de regressão logística multivariado (backward) para a determinação dos preditores independentes de eventos adversos, sendo representados por odds ratio (OR) e seu respectivo intervalo de confiança de 95\% (IC 95\%). Considerou-se significante $p<0,05$.

A análise estatística foi realizada com o software SPSS Inc. versão 13.0 (Chicago, Illinois, Estados Unidos).

\section{RESULTADOS}

As características demográficas e clínicas estão apresentadas na Tabela 1. Obteve-se informação de todos os pacientes e o seguimento clínico médio foi de 20,9 \pm 10,5 meses, com mediana de 21,9 meses $(13,5-29,2)$. Todos os eventos foram adjudicados e analisados por dois cardiologistas intervencionistas. As características angiográficas e do procedimento encontram-se na Tabela 2. A taxa de sucesso angiográfico imediato foi de $92 \%$. Os eventos intra-hospitalares e os ocorridos durante o seguimento clínico estão demonstrados, de forma cumulativa, na Tabela 3. A incidência de eventos adversos combinados foi de 11,5\% e a principal causa de óbito foi o choque cardiogênico $(3,8 \%)$. Durante o acompanhamento, detectou-se um único óbito comprovadamente não-cardíaco (traumatismo cranioencefálico com marcadores cardíacos negativos e eletrocardiograma sem novas alterações). Dois $(1,9 \%)$ pacientes apresentaram trombose do stent e necessitaram realizar nova RVA por angioplastia. Um único $(0,9 \%)$ caso apresentou reestenose clínica, sendo tratado por revascularização cirúrgica. Reinfarto ocor-
TABELA 1

Características demográficas e clínicas

\begin{tabular}{lc}
\hline Variáveis & $\mathbf{n}=\mathbf{1 0 4}$ \\
\hline Idade, anos & $63 \pm 12$ \\
Sexo masculino, \% & 82 \\
Hipertensão, \% & 63 \\
Diabetes, \% & 23 \\
DMNID & 19 \\
DMID & 4 \\
Tabagismo atual, \% & 41 \\
Dislipidemia, \% & 31 \\
Antecedente familiar, \% & 30 \\
Índice de massa corpórea, kg/m² & $26 \pm 4$ \\
Pressão arterial sistólica, mmHg & $134 \pm 30$ \\
Pressão arterial diastólica, mmHg & $84 \pm 20$ \\
Freqüência cardíaca, bpm & $79 \pm 17$ \\
Killip III ou IV à admissão, \% & 17 \\
PCR à admissão, \% & 3 \\
Multiarterial, \% & 67 \\
Angioplastia de resgate, \% & 4 \\
Fração de ejeção do VE, \% & \\
< 35 & 23 \\
$35-45$ & 294 \\
$46-50$ & \\
> 50 & \\
Pico enzimático (CK-MB massa), UI/ml & 20470 \\
\hline $\mathrm{n}=$ número de pacientes; DMNID = diabetes melito não- \\
insulino-dependente; DMID = diabetes melito insulino-de- \\
pendente; PCR = parada cardiorrespiratória; VE = ventrículo \\
esquerdo. \\
\hline
\end{tabular}

reu em 5 (4,8\%) pacientes: 2 relacionados à trombose do stent e tratados por intervenção coronária percutânea, 1 em decorrência de restenose e 2 relacionados à progressão da doença arterial coronária em outros vasos. As curvas de sobrevida livre de eventos adversos combinados e de RVA encontram-se representadas nas Figuras 1 e 2, respectivamente.

\section{Trombose e uso do clopidogrel}

Durante o período do estudo, detectaram-se 2 (1,9\%) casos de trombose de stent com comprovação angiográfica, classificados, por conseguinte, como tromboses definitivas. Um caso ocorreu no $5^{\text {o }}$ dia (trombose precoce, subaguda) e o outro no $363^{\circ}$ dia (trombose tardia). O primeiro caso ocorreu em paciente alérgica à aspirina e em uso de clopidogrel. No segundo caso, a paciente não estava em uso de clopidogrel. Ambos manifestaram-se como reinfarto e foram tratados por angioplastia, com óbito no caso da trombose precoce. Não foram documentados casos de trombose muito tardia. Um paciente faleceu, em circunstâncias não definidas, 35 dias após o procedimento, sendo esse evento 
TABELA 2

Características angiográficas e do procedimento

\begin{tabular}{|c|c|}
\hline Variáveis & $n=104$ \\
\hline \multicolumn{2}{|l|}{ Artéria relacionada ao infarto, $\%$} \\
\hline TCE & 1 \\
\hline $\mathrm{DA}$ & 45 \\
\hline $\mathrm{CX}$ & 14 \\
\hline CD & 36 \\
\hline PVS & 4 \\
\hline Uso de abciximab, \% & 72 \\
\hline Bifurcação, \% & 9 \\
\hline Trombo, \% & 70 \\
\hline Calcificação angiográfica, \% & 10 \\
\hline Stent direto, \% & 34 \\
\hline \multicolumn{2}{|l|}{ Fluxo TIMI pré-intervenção, \% } \\
\hline 0 ou 1 & 68 \\
\hline 2 & 12 \\
\hline 3 & 20 \\
\hline \multicolumn{2}{|l|}{ Fluxo TIMI pós-intervenção, \% } \\
\hline 0 ou 1 & 0 \\
\hline 2 & 8 \\
\hline 3 & 92 \\
\hline Comprimento da lesão, mm & $16,1 \pm 8,0$ \\
\hline $\begin{array}{l}\text { Diâmetro de referência } \\
\text { pré-intervenção, mm }\end{array}$ & $2,80 \pm 0,63$ \\
\hline $\begin{array}{l}\text { Diâmetro de referência } \\
\text { pós-intervenção, mm }\end{array}$ & $2,89 \pm 0,64$ \\
\hline $\begin{array}{l}\text { Diâmetro luminal mínimo } \\
\text { pré-intervenção, mm }\end{array}$ & $0,39 \pm 0,65$ \\
\hline $\begin{array}{l}\text { Diâmetro luminal mínimo } \\
\text { pós-intervenção, mm }\end{array}$ & $2,40 \pm 0,53$ \\
\hline Diâmetro da estenose pré-intervenção, \% & $87,6 \pm 18,5$ \\
\hline Diâmetro da estenose pós-intervenção, \% & $18,2 \pm 9,4$ \\
\hline \multicolumn{2}{|l|}{ Stents utilizados, \% } \\
\hline Cypher & 49 \\
\hline Taxus & 43 \\
\hline CoStar & 8 \\
\hline Stent/paciente & 1,3 \\
\hline Pressão de liberação, atm & $17 \pm 3$ \\
\hline TCA, s & $284 \pm 47$ \\
\hline Trombectomia, \% & 3 \\
\hline
\end{tabular}

$\mathrm{n}=$ número de pacientes; $\mathrm{TCE}=$ tronco de coronária esquerda; $D A=$ descendente anterior; $C X=$ circunflexa; $C D=$ coronária direita; TCA = tempo de coagulação ativado.

classificado como trombose possível. O tempo médio de uso de clopidogrel nessa população foi de 13,29 \pm 9,26 meses, considerando-se a informação de $72,2 \%$ dos pacientes. Os demais não souberam informar o tempo de uso da medicação $(21,1 \%)$ ou relataram nunca tê-la usado $(6,7 \%)$.

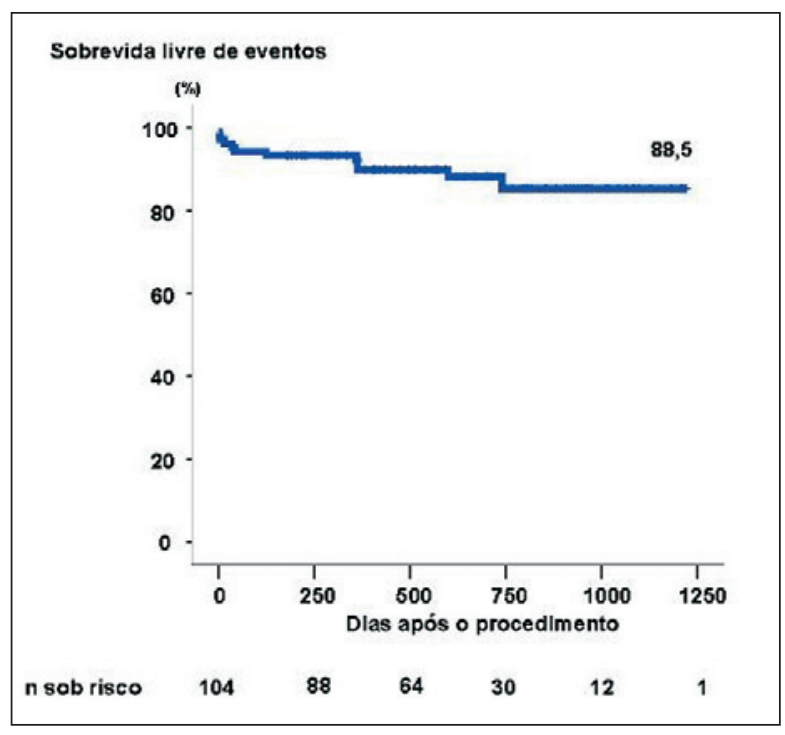

Figura 1 - Curva de Kaplan-Meier da sobrevida livre de eventos adversos maiores (\%).

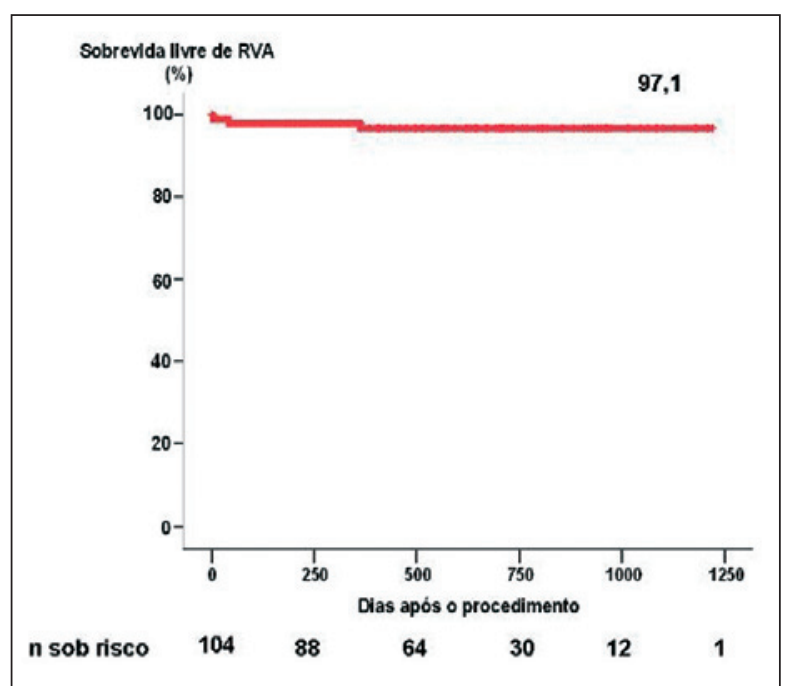

Figura 2 - Curva de Kaplan-Meier da sobrevida livre de revascularização do vaso-alvo (RVA) (\%).

\section{Preditores de eventos cardíacos maiores}

Na análise univariada, representada na Tabela 4, foram testadas as características clínicas, angiográficas e do procedimento, tendo sido identificadas a pressão arterial sistólica, a classificação de Killip, a doença coronária multiarterial, a fração de ejeção do ventrículo esquerdo e o uso de abciximab como variáveis relacionadas à ocorrência de eventos clínicos adversos. Essas variáveis, ajustadas no modelo de regressão logística multivariado (Figura 3), determinaram como preditor independente de evento adverso maior a presença da classificação Killip $\geq$ III à admissão (OR, 9,4; IC 95\%, $2,16-40,81 ; p<0,003)$. 
TABELA 3

Eventos clínicos intra-hospitalares e cumulativos

\begin{tabular}{|c|c|c|}
\hline Evento & Intra-hospitalar & Cumulativo $(20,9 \pm 10,5$ meses $)$ \\
\hline Eventos adversos combinados (\%) & $4(3,8)$ & $12(11,5)$ \\
\hline Óbito global (\%) & $4(3,8)$ & $7(6,7)$ \\
\hline Óbito cardíaco (\%) & $4(3,8)$ & $6(5,8)$ \\
\hline Reinfarto (\%) & $1(0,9)$ & $5(4,8)$ \\
\hline Revascularização do vaso-alvo (\%) & $1(0,9)$ & $3(2,9)$ \\
\hline Por trombose & $1(0,9)$ & $2(1,9)$ \\
\hline Por reestenose & - & $1(0,9)$ \\
\hline Trombose (\%) & $1(0,9)$ & $3(2,9)$ \\
\hline Definitiva & $1(0,9)$ & $2(1,9)$ \\
\hline Provável & $0(0,0)$ & $0(0,0)$ \\
\hline Possível & $0(0,0)$ & $1(0,9)$ \\
\hline \multicolumn{3}{|l|}{ Tempo da trombose } \\
\hline Precoce & $1(0,9)$ & $1(0,9)$ \\
\hline Tardia & - & $2(1,9)$ \\
\hline Muito tardia & - & $0(0,0)$ \\
\hline
\end{tabular}

TABELA 4

Preditores univariados de eventos cardíacos maiores

\begin{tabular}{lccc}
\hline Variável & Odds ratio & IC $\mathbf{9 5} \%$ & $\mathbf{p}$ \\
\hline Sexo & 1,58 & $0,38-6,50$ & 0,52 \\
Idade & 1,00 & $0,96-1,06$ & 0,71 \\
Hipertensão & 0,38 & $0,07-1,91$ & 0,24 \\
Diabetes & 0,59 & $0,16-2,19$ & 0,43 \\
Tabagismo & 2,02 & $0,50-8,15$ & 0,32 \\
FC & 1,01 & $0,98-1,05$ & 0,29 \\
PAS & 0,97 & $0,94-0,99$ & 0,03 \\
Multiarterial & 0,16 & $0,02-1,31$ & 0,08 \\
Trombo & 0,32 & $0,04-2,68$ & 0,29 \\
Abciximab & 0,13 & $0,02-1,63$ & 0,20 \\
Killip $\geq$ III & 0,15 & $0,04-0,54$ & $<0,01$ \\
Fração de ejeção do VE & 0,93 & $0,86-0,99$ & 0,02 \\
TIMl pós-procedimento & $0,33-13,0$ & 0,32 \\
\hline IC 95\% = intervalo de confiança de 95\% FC $=$ freqüência cardíaca: PAS = pressão arterial sistólica· VE = ventrículo esquerdo.
\end{tabular}

\section{DISCUSSÃO}

O presente estudo documenta os resultados imediatos e tardios de uma série consecutiva, não selecionada, de pacientes tratados na fase aguda do infarto do miocárdio com supradesnivelamento do segmento ST por angioplastia primária ou de resgate, com o emprego de stents farmacológicos. Dentre os resultados, documentou-se a ocorrência de uma única reestenose clínica e taxas de trombose em consonância com aquelas reportadas em estudos randomizados ${ }^{1,2,10}$. Destacase, também, o tempo de seguimento clínico do presente estudo, com mediana de 21,9 meses, superior ao seguimento dos estudos randomizados e registros publicados anteriormente ${ }^{1,2,10-12}$. Esses aspectos positivos do presente estudo são extremamente importantes, pois fornecem dados sobre a eficácia e a segurança a longo prazo dos stents farmacológicos utilizados no infarto do miocárdio, com uma população representativa da prática médica diária, do mundo real, diferindo, significativamente, do cenário menos complexo dos estudos randomizados. Nesse contexto, documentou-se praticamente a extinção da reestenose clínica com o emprego dos stents farmacológicos no infarto, resultado 


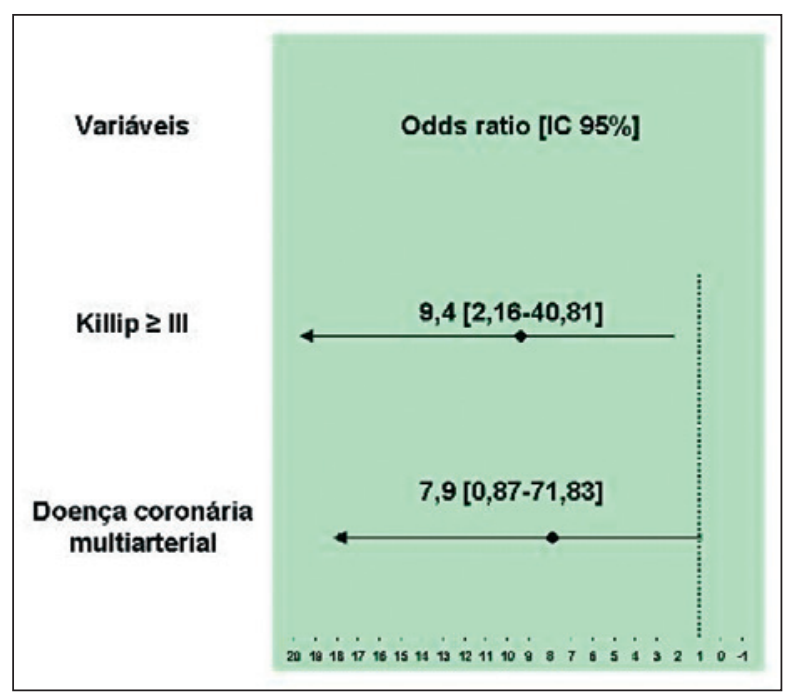

Figura 3 - Preditores multivariados de eventos adversos maiores.

que está de acordo com estudos randomizados com o emprego de stents farmacológicos ${ }^{1-3}$, porém incluindo lesões e pacientes de maior complexidade, como, por exemplo, choque cardiogênico, enxertos venosos, bifurcações, lesões longas, lesões com alta carga de trombo e lesões calcificadas, que foram critérios de exclusão desses estudos. Há evidências indicando que também os stents convencionais apresentam taxas de reestenose clínica relativamente baixas (cerca de 10\%) quando implantados no infarto agudo do miocárdio. Entretanto, a maioria dessas informações deriva de estudos realizados em populações de baixa complexidade tanto clínica como anatômica ${ }^{13-18}$. É provável que em cenários mais complexos esses excelentes resultados não se reproduzam, tornando mais evidente a vantagem dos stents farmacológicos. Esse é um aspecto importante, que merece ser analisado criteriosamente no momento da angioplastia primária, quando se faz a opção pelo emprego de stents farmacológicos ou convencionais.

Em relação à segurança, o presente estudo demonstrou taxas de trombose semelhantes àquelas dos estudos randomizados ${ }^{1,2,10}$ e de meta-análise recentemente publicada ${ }^{19}$, com a ausência de fenômenos trombóticos no seguimento muito tardio (> 1 ano). Esses resultados devem-se, provavelmente, ao tempo prolongado de uso do clopidogrel neste registro, que, em média, superou um ano. De fato, dados do registro PREMIER demonstram que pacientes tratados com stents farmacológicos no infarto agudo, e que interromperam precocemente (<30 dias) o uso dos tienopiridínicos, apresentaram mortalidade mais elevada em comparação aos pacientes que tiveram maior aderência ao uso desses medicamentos (7,5\% vs. 0,7\%; $p<0,0001)^{5}$. Esses dados despertaram a preocupação da comunidade cardiológica em relação à segurança dos stents farmacológicos utilizados no infarto. As justificativas e os fundamentos para essa preocupação são a maior propensão para ocorrência de eventos trombóticos no infarto, principalmente em lesões com carga trombótica acentuada $^{4}$ e em lesões bifurcadas ${ }^{20}$, em decorrência do atraso na reendotelização produzido pelos stents farmacológicos ${ }^{21}$ e da maior probabilidade de ocorrência de má aposição tardia das hastes dos stents ${ }^{22-24}$. Por tudo isso e diante dos achados desta investigação, os autores deste trabalho julgam imprescindível a manutenção do esquema de antiagregação plaquetária dupla por, no mínimo, 12 meses, quando do implante dos stents farmacológicos no infarto. Nos pacientes de baixo risco de sangramento, talvez se justifique a manutenção desse esquema por período ainda mais prolongado. O problema relacionado a essa recomendação reside na constatação de que o paciente com infarto agudo do miocárdio é, em geral, um "desconhecido" para o intervencionista, sendo, portanto, difícil garantir a aderência prolongada ao esquema antiplaquetário duplo. Em caso de dúvida, o mais apropriado seria o implante do stent convencional, já que as prioridades no tratamento do paciente com infarto devem ser, sempre, a segurança do paciente e o restabelecimento do fluxo coronário anterógrado normal, deixando-se o problema da reestenose em segundo plano. Entretanto, nas situações em que se pode garantir a aderência prolongada ao clopidogrel, o presente estudo demonstra que o emprego dos stents farmacológicos no infarto é uma estratégia segura e eficaz, em especial nas situações de maior complexidade, podendo, virtualmente, eliminar o fenômeno da reestenose e, conseqüentemente, a necessidade de nova RVA.

Por fim, embora o presente estudo tenha a grande virtude de representar o "mundo real" e estender o seguimento clínico além do primeiro ano, reconhecese a existência de diversas limitações inerentes a um registro retrospectivo, com número limitado de pacientes. Por esse motivo, é fundamental que os achados deste estudo sejam confirmados por outros estudos maiores, como, por exemplo, o HORIZONS, especialmente desenhados para responder, de forma definitiva, às questões sobre a segurança e a eficácia dos stents farmacológicos implantados durante a fase aguda do infarto agudo do miocárdio.

\section{REFERÊNCIAS BIBLIOGRÁFICAS}

1. Spaulding C, Henry P, Teiger E, Beatt K, Bramucci E, Carrié D, et al. Sirolimus-eluting versus uncoated stents in acute myocardial infarction. N Engl J Med. 2006;355(11):1093-104.

2. Menichelli M, Parma A, Pucci E, Fiorilli R, De Felice F, Nazzaro M, et al. Randomized trial of Sirolimus-Eluting Stent Versus Bare-Metal Stent in Acute Myocardial Infarction (SESAMI). J Am Coll Cardiol. 2007;49(19):1924-30.

3. Valgimigli M, Percoco G, Malagutti P, Campo G, Ferrari F, Barbieri D, et al. Tirofiban and sirolimus-eluting stent $v s$ abciximab and bare-metal stent for acute myocardial infarction: a randomized trial. JAMA. 2005;293(17):2109-17.

4. Sianos G, Papafaklis MI, Daemen J, Vaina S, van Mieghem 
CA, van Domburg RT, et al. Angiographic stent thrombosis after routine use of drug-eluting stents in ST-segment elevation myocardial infarction: the importance of thrombus burden. J Am Coll Cardiol. 2007;50(7):573-83.

5. Spertus JA, Kettelkamp R, Vance C, Decker C, Jones PG, Rumsfeld JS, et al. Prevalence, predictors, and outcomes of premature discontinuation of thienopyridine therapy after drug-eluting stent placement: results from the PREMIER registry. Circulation. 2006;113(24):2803-9.

6. Zhu MM, Feit A, Chadow H, Alam M, Kwan T, Clark LT. Primary stent implantation compared with primary balloon angioplasty for acute myocardial infarction: a meta-analysis of randomized clinical trials. Am J Cardiol. 2001;88(3):297-301.

7. Cutlip DE, Windecker S, Mehran R, Boam A, Cohen DJ, van Es GA, et al. Clinical end points in coronary stent trials: a case for standardized definitions. Circulation. 2007;115(17): 2344-51.

8. Gardin JM, Adams DB, Douglas PS, Feigenbaum $\mathrm{H}$, Forst $\mathrm{DH}$, Fraser $\mathrm{AG}$, et al. Recommendations for a standardized report for adult transthoracic echocardiography: a report from the American Society of Echocardiography's Nomenclature and Standards Committee and Task Force for a Standardized Echocardiography Report. J Am Soc Echocardiogr. 2002;15(3): 275-90.

9. Reiber JH, Serruys PW, Kooijman CJ, Wijns W, Slager CJ, Gerbrands JJ, et al. Assessment of short-, medium-, and long-term variations in arterial dimensions from computerassisted quantitation of coronary cineangiograms. Circulation. 1985;71(2):280-8.

10. Laarman GJ, Suttorp MJ, Dirksen MT, van Heerebeek L, Kiemeneij F, Slagboom T, et al. Paclitaxel-eluting versus uncoated stents in primary percutaneous coronary intervention. N Engl J Med. 2006;355(11):1105-13.

11. Lemos PA, Saia F, Hofma SH, Daemen J, Ong AT, Arampatziz $\mathrm{CA}$, et al. Short- and long-term clinical benefit of sirolimuseluting stents compared to conventional bare stents for patients with acute myocardial infarction. J Am Coll Cardiol. 2004;43(4):704-8.

12. Zhang F, Ge J, Qian J, Fan B, Wang Q, Ge L, et al. Sirolimus-eluting stents in real-world patients with ST segment elevation acute myocardial infarction. Int Heart J. 2007;48(3):303-11.

13. Suryapranata $H$, van't Hof AW, Hoorntje JC, de Boer MJ, Zijlstra F. Randomized comparison of coronary stenting with balloon angioplasty in selected patients with acute myocardial infarction. Circulation. 1998;97(25):2502-5.

14. Antoniucci D, Santoro GM, Bolognese L, Valenti R, Trapani M, Fazzini PF. A clinical trial comparing primary stenting of the infarct-related artery with optimal primary angioplasty for acute myocardial infarction: results from the Florence Randomized Elective Stenting in Acute Coronary Occlusions (FRESCO) trial. J Am Coll Cardiol. 1998;31(6):1234-9.

15. Rodriguez A, Bernardi V, Fernández M, Mauvecín C, Ayala $\mathrm{F}$, Santaera $\mathrm{O}$, et al. In-hospital and late results of coronary stents versus conventional balloon angioplasty in acute myocardial infarction (GRAMI trial). Gianturco-Roubin in Acute Myocardial Infarction. Am J Cardiol. 1998;81(11):1286-91.

16. Grines CL, Cox DA, Stone GW, Garcia E, Mattos LA, Giambartolomei A, et al. Coronary angioplasty with or without stent implantation for acute myocardial infarction. Stent Primary Angioplasty in Myocardial Infarction Study Group. N Engl J Med. 1999;341(26):1949-56.

17. Saito S, Hosokawa G, Tanaka S, Nakamura S. Primary stent implantation is superior to balloon angioplasty in acute myocardial infarction: final results of the primary angioplasty versus stent implantation in acute myocardial infarction (PASTA) trial. PASTA Trial Investigators. Catheter Cardiovasc Interv. 1999;48(3):262-8.

18. Stone GW, Grines CL, Cox DA, Garcia E, Tcheng JE, Griffin $\mathrm{J}$, et al. Comparison of angioplasty with stenting, with or without abciximab, in acute myocardial infarction. $N$ Engl J Med. 2002;346(13):957-66.

19. Pasceri V, Patti G, Speciale G, Pristipino C, Richichi G, Di Sciascio G. Meta-analysis of clinical trials on use of drugeluting stents for treatment of acute myocardial infarction. Am Heart J. 2007;153(5):749-54.

20. Ong AT, Hoye A, Aoki J, van Mieghem CA, Rodriguez Granillo GA, Sonnenschein K, et al. Thirty-day incidence and six-month clinical outcome of thrombotic stent occlusion after bare-metal, sirolimus, or paclitaxel stent implantation. J Am Coll Cardiol. 2005;45(6):947-53.

21. Virmani R, Farb A, Kolodgie FD. Histopathologic alterations after endovascular radiation and antiproliferative stents: similarities and differences. Herz. 2002;27(1):1-6.

22. Hong MK, Mintz GS, Lee CW, Park DW, Park KM, Lee BK, et al. Late stent malapposition after drug-eluting stent implantation: an intravascular ultrasound analysis with long-term follow-up. Circulation. 2006;113(3):414-9.

23. Ako J, Morino Y, Honda Y, Hassan A, Sonoda S, Yock PG, et al. Late incomplete stent apposition after sirolimus-eluting stent implantation: a serial intravascular ultrasound analysis. J Am Coll Cardiol. 2005;46(6):1002-5.

24. Siqueira DA, Abizaid AA, Costa JR, Feres F, Mattos LA, Staico $R$, et al. Late incomplete apposition after drugeluting stent implantation: incidence and potential for adverse clinical outcomes. Eur Heart J. 2007;28(11):1304-9. 